\title{
The EGPs: The Eclosion Hormone and Cyclic GMP-Regulated Phosphoproteins. II. Regulation of Appearance by the Steroid Hormone 20-Hydroxyecdysone in Manduca sexta
}

\author{
David B. Morton and James W. Truman \\ Department of Zoology, University of Washington, Seattle, Washington 98195
}

\begin{abstract}
Two proteins (the EGPs) in the CNS of the tobacco hornworm Manduca sexta are phosphorylated by the action of the neuropeptide eclosion hormone (EH), which triggers ecdysis behavior (Morton and Truman, 1986, 1988). The onset of sensitivity to EH requires prior exposure to the steroid hormone 20-hydroxyecdysone (20-HE). A series of steroid removal and replacement experiments indicates that the EGPs are also regulated by $20-\mathrm{HE}$ with a time course that is similar to that seen for the 20-HE regulation of behavioral sensitivity to EH. This suggests that the steroid regulation of EH sensitivity is due to the regulation of the EGPs. The appearance of the EGPs requires not only the presence of 20-HE, but also its subsequent removal. The appearance of the EGPs can be blocked in vivo and in vitro by maintaining artificially elevated levels of 20-HE, but only up to a particular time in development. The ending of this steroid-sensitive period occurs $4 \mathrm{hr}$ before the normal appearance of the EGPs, consistent with the hypothesis that the EGPs are synthesized de novo in response to the removal of 20-HE.
\end{abstract}

There are a number of peptide-mediated behaviors in mammals that require "priming" of the CNS by a prior exposure to steroid hormones. For example, the action of luteinizing hormone-releasing hormone (LHRH), which acts on the brain of rats to induce lordosis, requires the prior exposure of the brain to estrogen (Pfaff, 1973). Similarly, estrogen-priming facilitates the response of virgin female rats to oxytocin in the induction of "short-latency maternal behavior" (Fahrbach et al., 1985). A similar type of steroid-peptide interaction in invertebrates is the action of ecdysteroids and eclosion hormone $(\mathrm{EH})$ on the insect CNS. The neuropeptide EH triggers the stereotyped ecdysis behavior used to shed the old cuticle at the end of each molt in Lepidoptera (Truman, 1978). In the tobacco hornworm, Manduca sexta, the insect only responds to EH during a narrow temporal window that occurs late in each molt (Reynolds et al., 1979; Truman et al., 1980). This transient appearance is regulated by the steroid molting hormone 20 -hydroxyecdysone (20HE; Truman et al., 1983).

\footnotetext{
Received May 15, 1987; revised Aug. 10, 1987; accepted Sept. 10, 1987.
}

We wish to thank R. Booker, S. E. Fahrbach, J. Gabriel, K. A. Mesce, L. M. Riddiford, B. A. Trimmer, and J. L. Witten for comments on the manuscript and many helpful discussions during the course of this work. This work was supported by NIH Grant NS-13079.

Correspondence should be addressed to David B. Morton and James W. Truman, Department of Zoology, NJ-15, University of Washington, Seattle, WA 98195.

Copyright (C) 1988 Society for Neuroscience $0270-6474 / 88 / 041338-08 \$ 02.00 / 0$
In previous reports (Morton and Truman, 1986, 1988), we described 2 proteins in the CNS of Manduca that are phosphorylated by the action of EH - the EGPs (the eclosion hormoneand $c G M P$-regulated $p$ hosphoproteins). At pupal ecdysis, the EGPs can only be phosphorylated in vitro for a short time prior to ecdysis. This time coincides with EH sensitivity. The present paper explores the relationship of steroid exposure to the ability to phosphorylate the EGPs.

\section{Materials and Methods}

Rearing and staging of animals. Larvae of the tobacco hornworm Manduca sexta were reared individually on an artificial diet (Bell and Joachim, 1978) at $26^{\circ} \mathrm{C}$ under a 17L:7D photoperiod. At the end of the terminal (fifth) larval stage the insect ceased to feed, emptied its gut, and entered the "wandering" stage. Pupal ecdysis occurred $4 \mathrm{~d}$ later.

Timing of events prior to pupal ecdysis was made with reference to the pigmentation of a pair of sclerotized bars on the dorsal surface of the metathoracic segment (the "trace bars" stage), which occurred 24 $\mathrm{hr}$ before ecdysis at $22^{\circ} \mathrm{C}$. The resorption of molting fluid into the anterior segments (the "anterior shrink" stage) occurred $4 \mathrm{hr}$ before ecdysis (Truman et al., 1980).

Phosphorylation, separation, and visualization of the EGPS. Nervous tissue was removed and homogenized in $50 \mathrm{~mm}$ HEPES buffer, $5 \mathrm{~mm}$

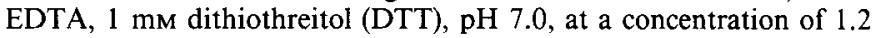
$\mathrm{mg}$ protein $/ \mathrm{ml}$ buffer. The phosphorylation was carried out by incubating $100 \mu$ lof the homogenate with $40 \mu 1250 \mathrm{~mm}$ HEPES buffer, $\mathrm{pH}$

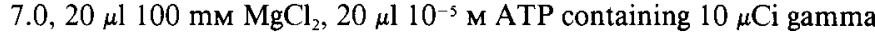
${ }^{32} \mathrm{P}-\mathrm{ATP}$ (New England Nuclear; approximately $3000 \mathrm{Ci} / \mathrm{mmol}$ ), and $20 \mu \mathrm{l}$ of a $1 \mathrm{~mm}$ solution of cGMP (final concentration, $0.1 \mathrm{~mm}$ ). The reaction was carried out at $30^{\circ} \mathrm{C}$ for $5 \mathrm{~min}$ and was started by the addition of the ATP and stopped with $200 \mu \mathrm{l} 20 \%$ trichloroacetic acid (TCA). The reaction mixture was allowed to stand on ice for $30 \mathrm{~min}$, centrifuged at $10,000 \times g$ for $5 \mathrm{~min}$ at $4^{\circ} \mathrm{C}$, and the pellet dissolved in $40 \mu \mathrm{l}$ sample buffer [9.5 м urea, 2\% Nonidet P40 (Sigma), 1.6\% Ampholines, pH 57, 0.4\% Ampholines, pH 3.5-10 (both LKB), 5\% 2-mercaptoethanol (2-ME), and $0.01 \%$ SDS]. The samples were left at room temperature overnight, vortexed, and centrifuged at $10,000 \times g$ for $5 \mathrm{~min}$ at $4^{\circ} \mathrm{C}$ and the supernatant separated by 2-dimensional SDS-PAGE.

The first dimension of separation was performed by nonequilibrium pH gradient electrophoresis (NEPHGE; O'Farrell et al., 1977), with gels containing $9.2 \mathrm{M}$ urea, $4 \%$ acrylamide, $2 \%$ Nonidet P40, and $2 \% \mathrm{Am}-$ pholines, $\mathrm{pH} 3.5-10$ (LKB). Samples of $40 \mu \mathrm{l}$ (approximately $120 \mu \mathrm{g}$ protein) were loaded onto the gels and run for $6 \mathrm{hr}$ at $500 \mathrm{~V}$, removed from the tubes and equilibrated for $1 \mathrm{hr}$ in $62.5 \mathrm{~mm}$ Tris- $\mathrm{HCl}, \mathrm{pH} 6.8$, $2.3 \%$ SDS, $10 \%$ glycerol, and 5\% 2-ME. The second dimension was separated using SDS-PAGE (Laemmli, 1970) on 10\% acrylamide gels containing $0.1 \%$ SDS, run at $50 \mathrm{~V}$ for $1 \mathrm{hr}$, then at $150 \mathrm{~V}$ until the tracking dye was $1 \mathrm{~cm}$ from the bottom of the gel. The gels were then silver-stained, as described by Merril et al. (1983), dried and exposed to preflashed Kodak XRP-1 film with DuPont Lightning Plus intensification screens. Exposure time was typically $4 \mathrm{~d}$. Molecular weight standards used were phosphorylase $B(97,400)$, bovine albumin $(66,000)$, egg albumin $(45,000)$, glyceraldehyde-3-phosphate dehydrogenase $(36,000)$, carbonic anhydrase $(29,000)$, and trypsinogen $(24,000)$ (all Sigma). Isoelectric point markers used were trypsin inhibitor (4.55), 
human carbonic anhydrase B (6.57), and myoglobin (6.76 and 7.16) (all Sigma).

In all of the fluorograms shown in the present paper, only a part of the fluorograms is shown. This portion covers the molecular weight range of $70-35 \mathrm{kDa}$ and the isoelectric point range of 5.0 (left) to 7.0 (right).

Ecdysteroid manipulations. Ecdysone is synthesized and secreted in the prothoracic glands, which are located in the thorax. The target tissues then convert the ecdysone to 20-HE (see O'Connor, 1985). When these 2 forms are not distinguished, the term "ecdysteroids" will be used.

Ligation of larvae or prepupae between the thorax and abdomen prevents exposure of the abdominal nervous system to the normal level of ecdysteroids. Ligations were performed by tightly tying silk thread ligatures between the thorax and abdomen of the insect and then removing the head and thorax. Abdomens isolated in this way were infused with 20-HE (Sigma) by inserting a length of polyethylene tubing, via the dorsal horn, into the hemocoel. The 20-HE was then pumped into the abdomen using a motor-driven syringe (Sage Instruments) at a rate of $5.4 \mu \mathrm{l} / \mathrm{hr}$ for $12 \mathrm{hr}$. The concentration of 20 -HE was $1 \mathrm{mg} / \mathrm{ml}$ and was checked spectrophotometrically at $240 \mathrm{~nm}\left(\mathrm{E}_{240}=12,670\right.$; Meltzer, 1971).

Isolated nervous system incubations. Abdominal nervous systems were dissected, leaving as much of the tracheal supply possible intact, and rinsed in Grace's insect medium (Gibco). They were then placed in 0.5 $\mathrm{ml}$ of Grace's medium in the presence or absence of 20-HE. The nervous systems were incubated at $26^{\circ} \mathrm{C}$ in a $95 \% \mathrm{O}_{2}-5 \% \mathrm{CO}_{2}$ atmosphere, removed after the alloted time, homogenized, and phosphorylated in the presence of $0.1 \mathrm{~mm} \mathrm{CGMP}$ and ${ }^{32} \mathrm{P}-\mathrm{ATP}$. The labeled proteins were then separated by 2-dimensional SDS-PAGE, as described above.

\section{Results}

\section{The ability to phosphorylate the EGPS requires $20-H E$}

The ecdysteroid titer present in the hemolymph during the $5 \mathrm{~d}$ before pupal ecdysis is shown in Higure $1 A$. Two peaks of ecdysteroid are seen; a small peak $5 \mathrm{~d}$ before pupal ecdysis and a larger one $2 \mathrm{~d}$ before ecdysis (Bollenbacher ct al., 1981). The small peak is known as the "commitment pulse" and "commits" the tissues to become pupal at the next molt. The second largest peak, known as the "prepupal peak," is responsible for pupal differentiation (see Riddiford, 1985).

The EGPs are first able to be phosphorylated in vitro $8 \mathrm{hr}$ before pupal ecdysis (Morton and Truman, 1986, 1988). The absence of the EGPs on fluorograms prior to this time could be due either to their absence or to their inability to accept labeled phosphate in vitro. As detailed in the Discussion, we favor the former hypothesis, but the alternate explanation cannot yet be ruled out. In describing the following results, we have equated the absence of incorporation of label with the absence of the proteins themselves.

The roles of the prepupal peak in the appearance of the EGPs was investigated by a series of manipulations that removed and rcplaced the ecdysteroids. By ligating the animals $4 \mathrm{~d}$ before ecdysis, the prepupal peak was eliminated (Fig. $1 B$ ). Such abdomens survived for over a week, but they did not give evidence for the production of a new pupal cuticle. When nervous systems from such abdomens were examined $4 \mathrm{~d}$ after ligation (a time coinciding with pupal ecdysis in control animals), no trace of the EGPs could be seen on fluorograms (Fig. $2 B$ ).

The prepupal peak was artificially replaced in ligated abdomens by infusing them with 20-HE for $12 \mathrm{hr}$, starting $24 \mathrm{hr}$ after ligation. This resulted in hemolymph levels of ecdysteroid very similar to those seen in intact animals (Fig. $1 C$; Morton and Truman, 1985). Accordingly, these abdomens produced a new pupal cuticle according to an approximately normal time schedule. The degree of tanning of the pupal cuticle is indicative of approaching ecdysis in intact animals (Truman et al., 1980). This marker was used to estimate the development of the infused

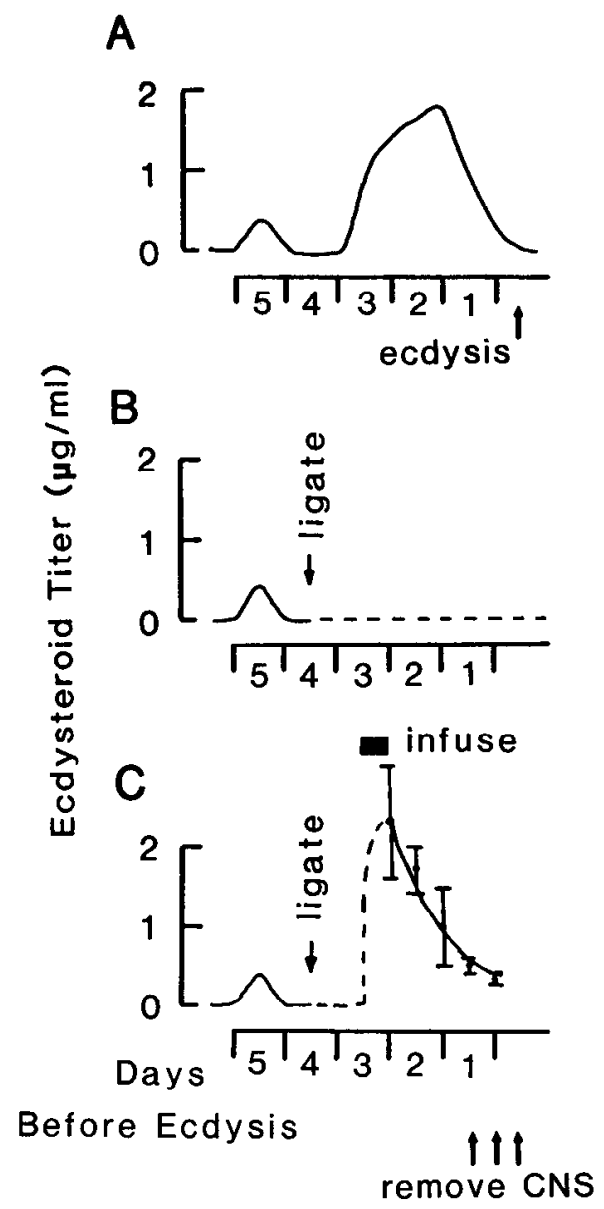

Figure 1. A, Schematic representation of hemolymph ecdysteroid levels during pupal development (based on data from Bollenbacher et al., 1981 , and the unpublished observations of L. M. Riddiford). $B$, Effect on the ecdysteroid levels of ligating animals between the thorax and abdomen $4 \mathrm{~d}$ before ecdysis. The dashed line represents the presumed lack of ecdysteroids in the hemolymph. $C$, Ecdysteroid levels after a 12 $\mathrm{hr}$ infusion of 20-HE. The dashed line represents the presumed increase in ecdysteroids during infusion, and the solid line shows data from Morton and Truman (1985), obtained by RIA, for the ecdysteroid titers at various times after termination of $20-\mathrm{HE}$ infusion.

abdomens. At $36 \mathrm{hr}$ after the end of the infusion, pupal cuticle could be seen under the larval cuticle, but there was very little, if any, tanning of this cuticle. At $48 \mathrm{hr}$ after infusion, fairly extensive tanning was visible on the new pupal cuticle. By 60 $\mathrm{hr}$ after infusion the animals fell into 2 distinct groups. About half showed a degree of tanning on the pupal cuticle that was very similar to that of animals just before pupal ecdysis, while the remainder of the animals showed much heavier tanning, analogous to that of animals after pupal ecdysis.

In vitro phosphorylation of nervous systems removed from abdomens $36 \mathrm{hr}$ after the end of the infusion showed no trace of the EGPs (Fig. 2C). By $48 \mathrm{hr}$, however, the EGPs were clearly visible on fluorograms (Fig. $2 D$ ). In the abdomens that were examined $60 \mathrm{hr}$ after infusion, the nervous systems of those that showed tanning similar to that seen in animals before ecdysis also showed the presence of the EGPs (Fig. 2E). By contrast, the other group, which had pupal cuticle characteristic of postecdysis animals, showed no incorporation of label into the EGPs (Fig. $2 F$ ). In intact animals there is also an abrupt loss of incorporation of ${ }^{32} \mathrm{P}$ into the EGPs in nervous systems removed 


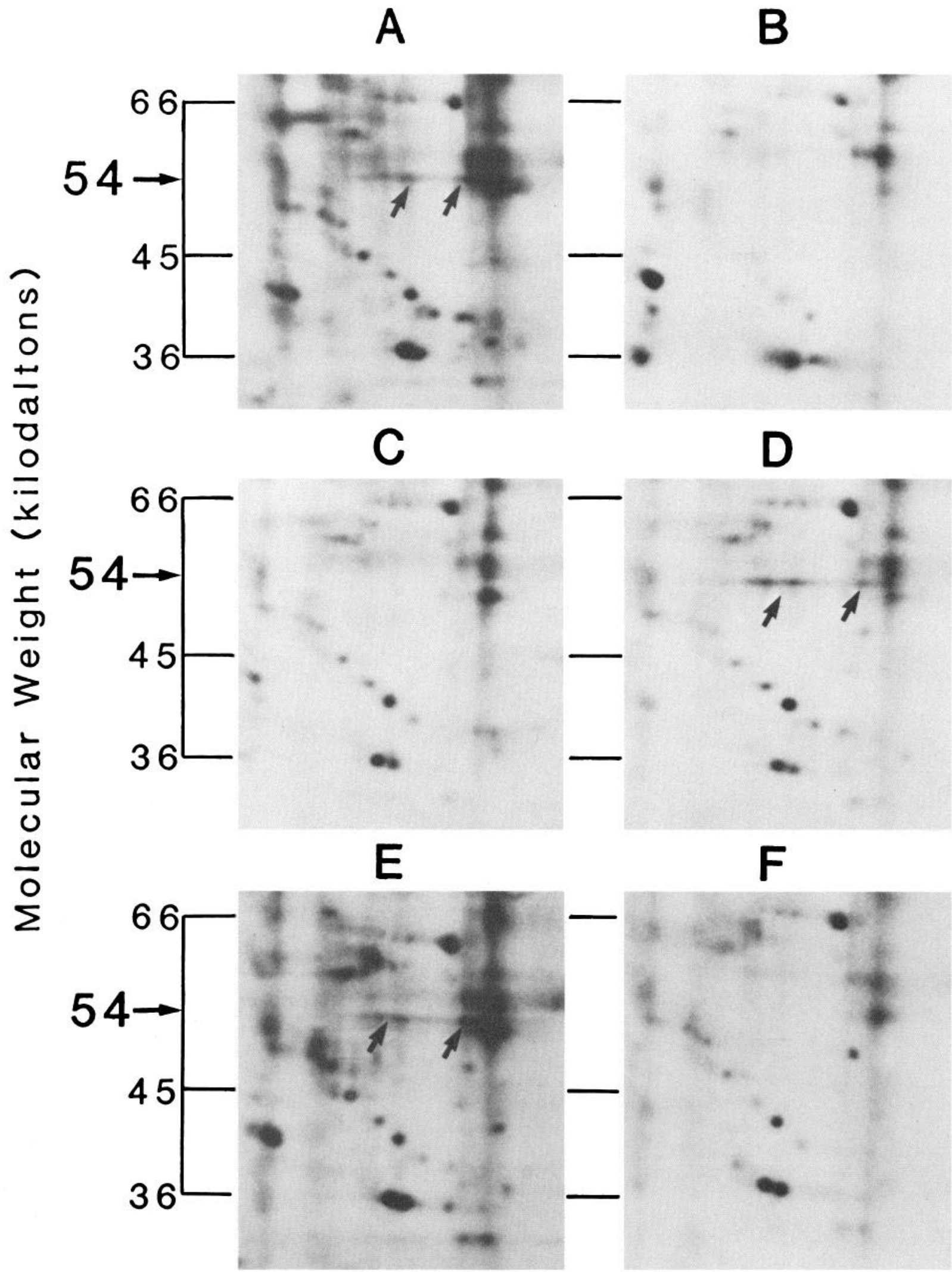

Figure 2. The prepupal peak of ecdysteroids is necessary in order for the EGPs to be phosphorylated. Abdominal nervous systems were removed from animals after a variety of treatments, homogenized, and phosphorylated with ${ }^{32} \mathrm{P}$-ATP in the presence of $0.1 \mathrm{mM} \mathrm{cGMP}$. The samples were then separated by 2D SDS-PAGE and fluorograms made. $A$, Nervous systems removed from intact animals $4 \mathrm{hr}$ before ecdysis. $B$, Nervous systems removed from abdomens that were ligated $4 \mathrm{~d}$ before ecdysis and then held for $4 \mathrm{~d}$. $C-F$, Nervous systems removed from abdomens ligated at the same time as in $B$ and infused with 20 -HE for $12 \mathrm{hr}$. The nervous systems were removed $36 \mathrm{hr}(C), 48 \mathrm{hr}(D)$, and $60 \mathrm{hr}(E, F)$ after the infusion. In $E$ the abdomens had pupal cuticle similar to that of intact animals before ecdysis, and in $F$ the abdomens had pupal cuticle similar to that of intact animals after ecdysis. Arrows show the presence of the EGPs. 


\section{A}

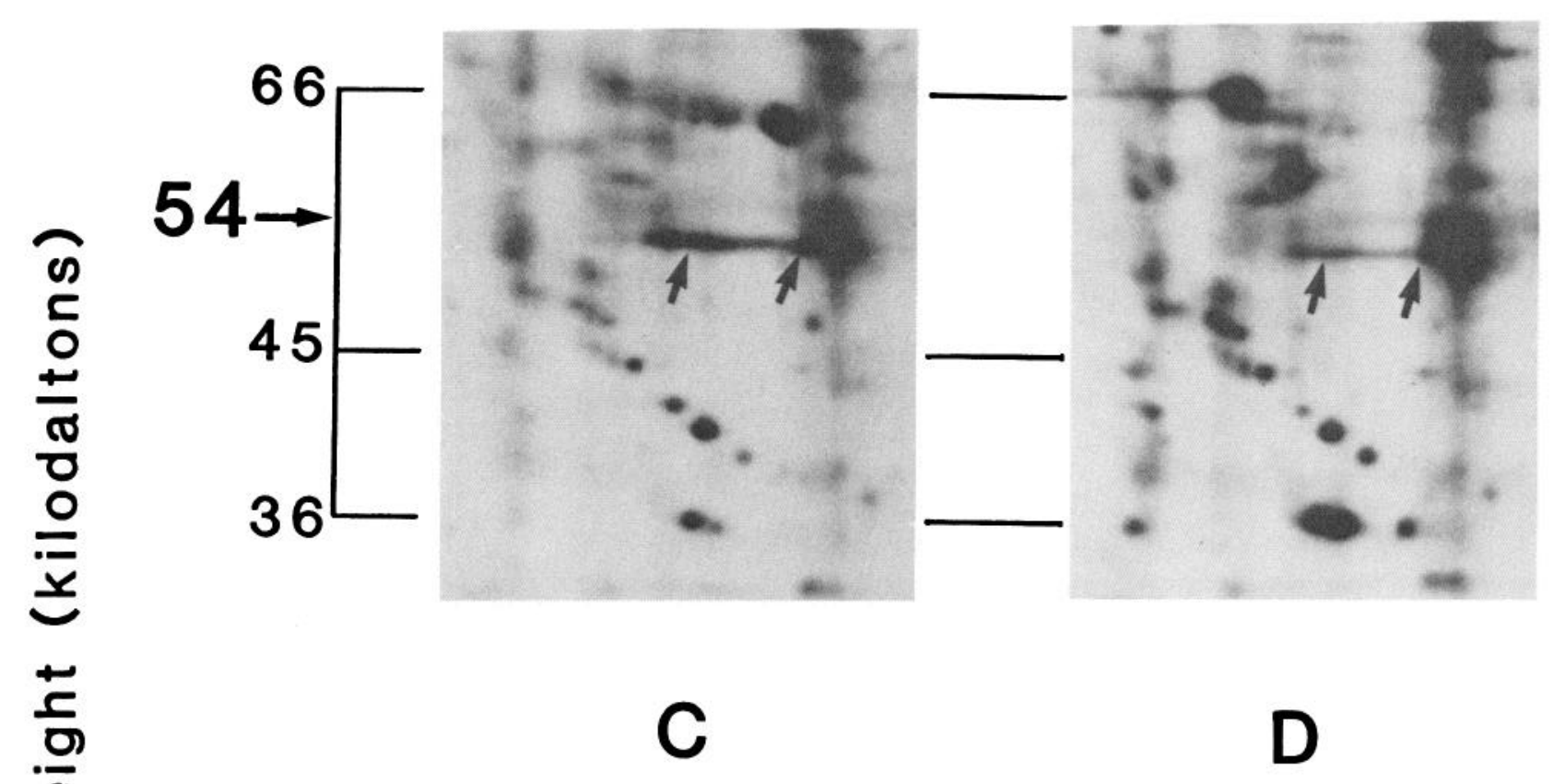

$\stackrel{1}{3}$

\begin{tabular}{l}
$\frac{\pi}{3}$ \\
0 \\
$\frac{0}{0}$ \\
\hline
\end{tabular}
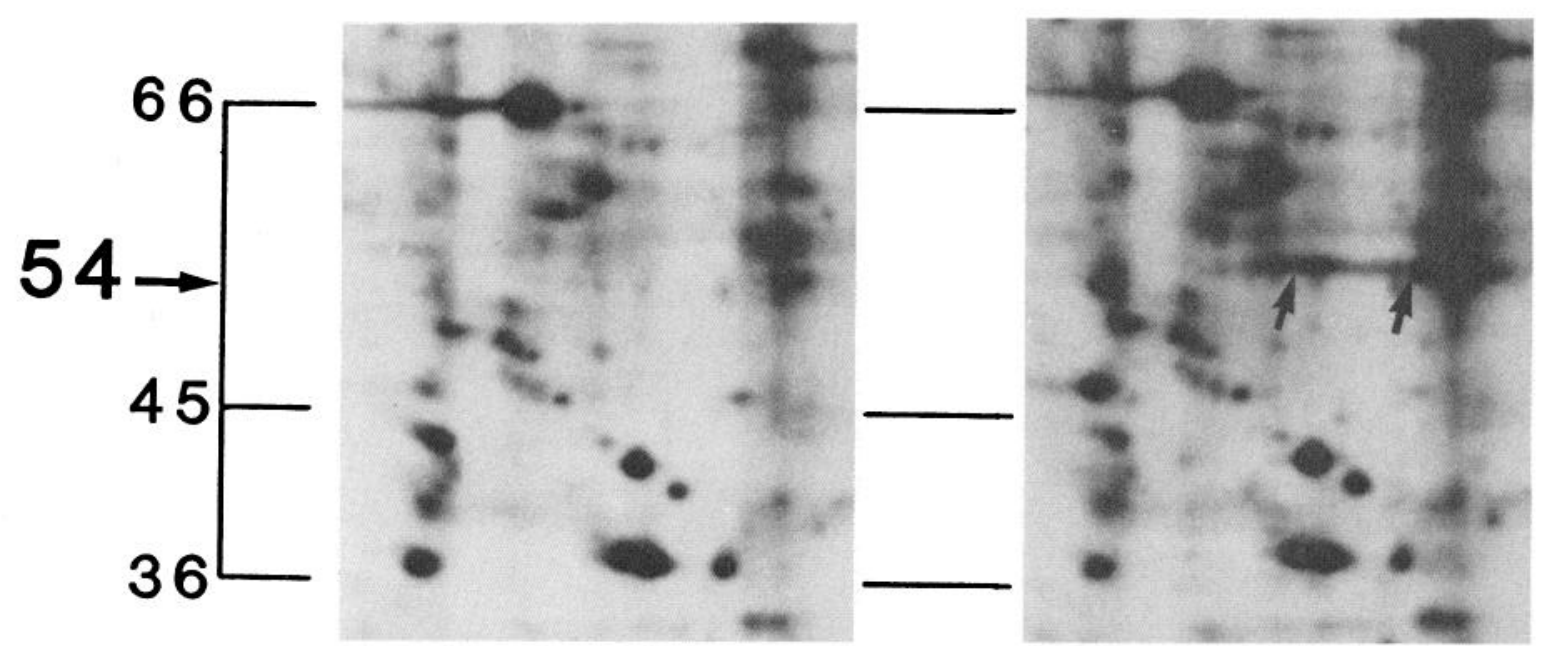

Figure 3. The appearance of the ability to phosphorylate the EGPs in cultured nervous tissue is blocked by the presence of 20-HE. A, Control. Nervous systems were removed from animals $4 \mathrm{hr}$ before ecdysis, homogenized, phosphorylated by ${ }^{32} \mathrm{P}-\mathrm{ATP}$ in the presence of $0.1 \mathrm{mM}$ cGMP, the proteins separated by 2D SDS-PAGE, and fluorograms made. $B-D$, The nervous systems were removed from animals $24 \mathrm{hr}$ before ecdysis and incubated for $24 \mathrm{hr}$ in the presence or absence of 20-HE before being homogenized, phosphorylated, and separated under the same conditions as in $A$. B, Incubations in the absence of 20 -HE. $C$, Incubations in the presence of $0.05 \mu \mathrm{g} / \mathrm{ml} 20-\mathrm{HE}$. $D$, Incubations in the presence of $0.01 \mu \mathrm{g} /$ $\mathrm{ml} 20$-HE. Arrows show the presence of the EGPs.

after pupal ecdysis (Morton and Truman, 1986, 1988). We suggest that in both intact and infused abdomens, this inability to label the EGPs in vitro is due to endogenously released EH stimulating the phosphorylation of the EGPs with unlabeled phosphate in vivo.

The length of ecdysteroid exposure necessary to induce the appearance of the EGPs on fluorograms was determined by infusing 20-HE into isolated abdomens at the rate of $5.4 \mu \mathrm{l} / \mathrm{hr}$ for various periods of time (Table 1). Sixty hours after the end of infusion, the nervous systems were removed, subjected to in vitro phosphorylation, and examined for their ability to phos- phorylate the EGPs. The EGPs were present after both a 6 and a $9 \mathrm{hr}$ infusion, but could not be phosphorylated if the infusion lasted for only $3 \mathrm{hr}$. The response of the nervous system to ecdysteroid infusion was similar to the pupal cuticle response of the epidermis. Abdomens infused for $3 \mathrm{hr}$ failed to synthesize pupal cuticle, whereas 6 and $9 \mathrm{hr}$ infusions were sufficient for the formation of pupal cuticle by $60 \mathrm{hr}$ after the end of infusion. Thus, under the infusion paradigm that we used, there appeared to be a minimum ecdysteroid exposure (between 3 and $6 \mathrm{hr}$ ) required for the expression of the ability to phosphorylate the EGPs. 
Figure 4. Timing of the end of the steroid-sensitive period in prepupal Manduca. Filled circles show the time course of normal development of EH responsiveness. Animals were taken at various times before pupal ecdysis and injected with 0.5 units of $\mathrm{EH}$. The number showing ecdysis behavior prematurely (within $2 \mathrm{hr}$ of the time of injection) was determined. Open circles show the effect of 20-HE in delaying this $\mathrm{EH}$ response. Animals were injected with 40 $\mu \mathrm{g}$ of 20 -HE at various times before ecdysis and left until $2 \mathrm{hr}$ before the normal time of ecdysis. They were then injected with 0.5 units of $\mathrm{EH}$, and the number responding with premature ecdysis bchavior was determined. Each point represents at least 8-10 animals. The time between the $50 \%$ points of each curve is $5 \mathrm{hr}$, as shown. The dashed line shows the ecdysteroid titer during this time, as measured by RIA (data from Truman et al., 1983).

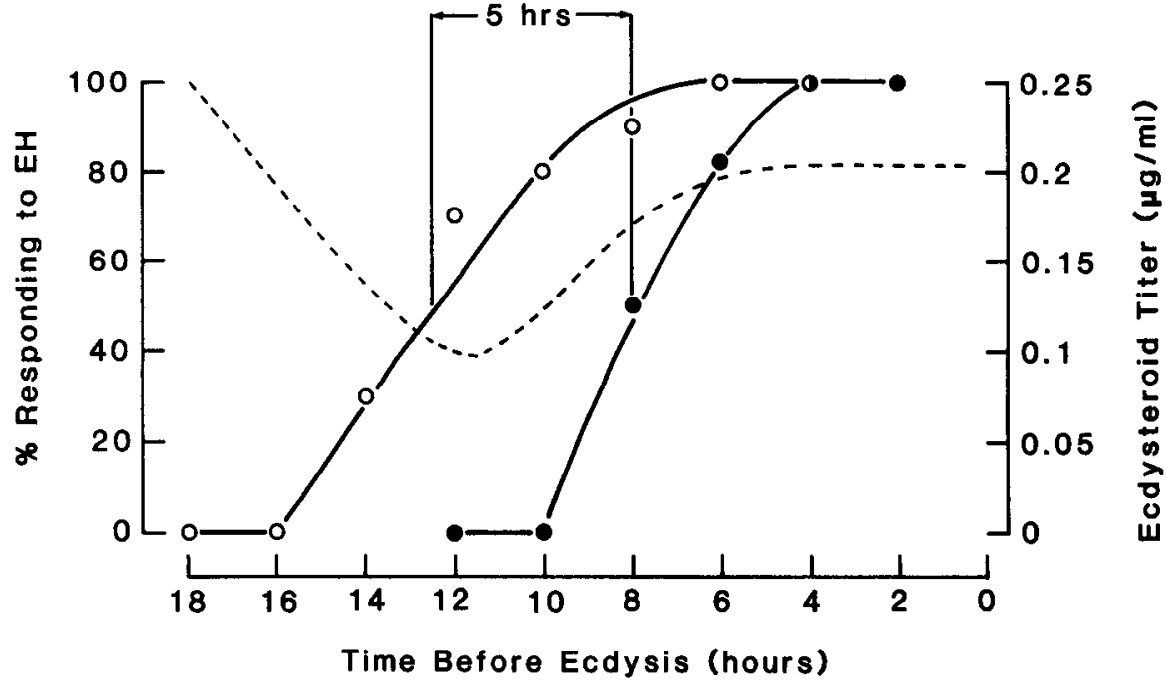

Table 1. Length of 20-HE exposure necessary to induce the ability to phosphorylate the EGPs in the abdominal CNS of Manduca

\begin{tabular}{|c|c|c|}
\hline $\begin{array}{l}\text { Infusion } \\
\text { time (hr) }\end{array}$ & $\begin{array}{l}\text { Total amount of } \\
20-\mathrm{HE}(\mu \mathrm{g})\end{array}$ & $\begin{array}{l}\text { Incorporation } \\
\text { of }{ }^{32} \mathrm{P} \text { into } \\
\text { EGPs }\end{array}$ \\
\hline 3 & 16 & - \\
\hline 6 & 33 & + \\
\hline 9 & 49 & + \\
\hline 12 & 65 & + \\
\hline
\end{tabular}

Animals were ligated and infused with $20-\mathrm{HE}$ at $5.4 \mu \mathrm{l} / \mathrm{hr}$ for various lengths of time. Sixty hours after the end of infusion, the abdominal CNS was removed, homogenized, and phosphorylated in the presence of ${ }^{32}$ P-ATP and $0.1 \mathrm{mM} \mathrm{CGMP.}$ The labeled proteins were separated hy 2D SDS-PAGE and fluorograms made and examined for the presence of the EGPs. +, Presence of the EGPs; -, no EGPs could be detected on the fluorograms.

\section{Relationship of the ability to phosphorylate the EGPS to the decline in ecdysteroids}

Although the appearance of ecdysteroids at a molt is necessary for the acquisition of EH sensitivity, the steroids must then be removed from the system to allow EH to act (Truman et al., 1983). We investigated whether the appearance of the EGPs was also subject to this requirement.

During the pupal molt, the ecdysteroid titer peaks at about 36-48 $\mathrm{hr}$ before pupal ecdysis (Bollenbacher et al., 1981). It then declines to a minimum value of $0.1 \mu \mathrm{g}$ of $20-\mathrm{HE} \mathrm{Eq} / \mathrm{ml}$ at 10 $12 \mathrm{hr}$ before ecdysis (Truman et al., 1983). At $24 \mathrm{hr}$ before ecdysis, the ecdysteroids are present at about $0.4 \mu \mathrm{g} 20-\mathrm{HE} \mathrm{Eq} /$ $\mathrm{ml}$ (Truman et al., 1983). The EGPs cannot be phosphorylated in vitro at this time (Morton and Truman, 1986, 1988). Abdominal nervous systems were removed from $-24 \mathrm{hr}$ prepupae and maintained in culture for $24 \mathrm{hr}$. When maintained in the absence of $20-\mathrm{HF}$, these nervous systems showed the presence of the EGPs at the end of the $24 \mathrm{hr}$ culture period (Fig. $3 B$ ). By contrast, when similar nervous systems were cultured in the presence of $0.05 \mu \mathrm{g} / \mathrm{ml}$ of 20-HE, the EGPs could not be phosphorylated in vitro (Fig. 3C). A lower level of 20-HE (0.01 $\mu \mathrm{g} /$ $\mathrm{ml}$ ) permitted the subsequent labeling of the EGPs (Fig. $3 D$ ). These data show that there is a critical minimum level to which the ecdysteroid titer must drop to permit expression of the ability to phosphorylate the EGPs. This level is mimicked in vitro by between 0.05 and $0.01 \mu \mathrm{g} / \mathrm{ml} 20-\mathrm{HE}$.

During the molt to the pupal stage, there is a critical period after which the application of exogenous 20 -HE no longer prevents the appearance of EH sensitivity (Truman et al., 1983). Two sets of experiments were performed, one to determine the time at which 20-HE no longer prevented EH from triggering ecdysis behavior, and the second to determinc the time at which 20-HE applications no longer prevented the subsequent ability to phosphorylate the EGPs.

The critical period for behavioral sensitivity was defined by injecting prepupae with $40 \mu \mathrm{g}$ of $20-\mathrm{HE}$ at various times after they entered the "trace bars" stage ( $24 \mathrm{hr}$ before pupal ecdysis). At $22 \mathrm{hr}$ after they entered this stage ( $2 \mathrm{hr}$ before ecdysis), the groups were challenged with a standard dosage of 0.5 units of EH. The number of animals that then ecdysed less than $2 \mathrm{hr}$ after injection was determined. Injection of $20-\mathrm{HE}$ at $16 \mathrm{hr}$ before ecdysis or earlier prevented the animals from aquiring EH sensitivity when assayed $2 \mathrm{hr}$ before the expected time of ecdysis. Injection of 20-HE $6 \mathrm{hr}$ before ecdysis was completely ineffective in delaying EH sensitivity (Fig. 4). The end of the steroid-sensitive period is defined as the time at which the EH sensitivity of $50 \%$ of the animals is unaffected by 20 -HE. Figure 4 shows that this occurred just over $12 \mathrm{hr}$ before ecdysis and 5 $\mathrm{hr}$ before $50 \%$ of the animals normally acquired EH sensitivity. It also coincided with the time when the hemolymph ecdysteroid levels fell to their minimum value $(10-12 \mathrm{hr}$ before ecdysis; Truman et al., 1983).

To assess the critical period when ecdysteroids inhibit the appearance of the EGPs, we injected groups of prepupae with $40 \mu \mathrm{g}$ of 20 -IIE at various times during pupal development. They were kept until $2 \mathrm{hr}$ before the normal time of ecdysis and their nervous systems were then removed and analyzed for the incorporation of ${ }^{32} \mathrm{P}$ into the EGPs. Figure 5 shows that injecting animals with $20-\mathrm{HE}$ at 14 or $12 \mathrm{hr}$ before ecdysis blocked the ability of the EGPs to incorporate ${ }^{32} \mathrm{P}$-phosphate. At $10 \mathrm{hr}$ before ecdysis, 20-HE treatment resulted in the weak, but definite presence of the EGPs on the fluorograms. Densitometer scans of the fluorograms (see Morton and Truman, 1988) from animals 

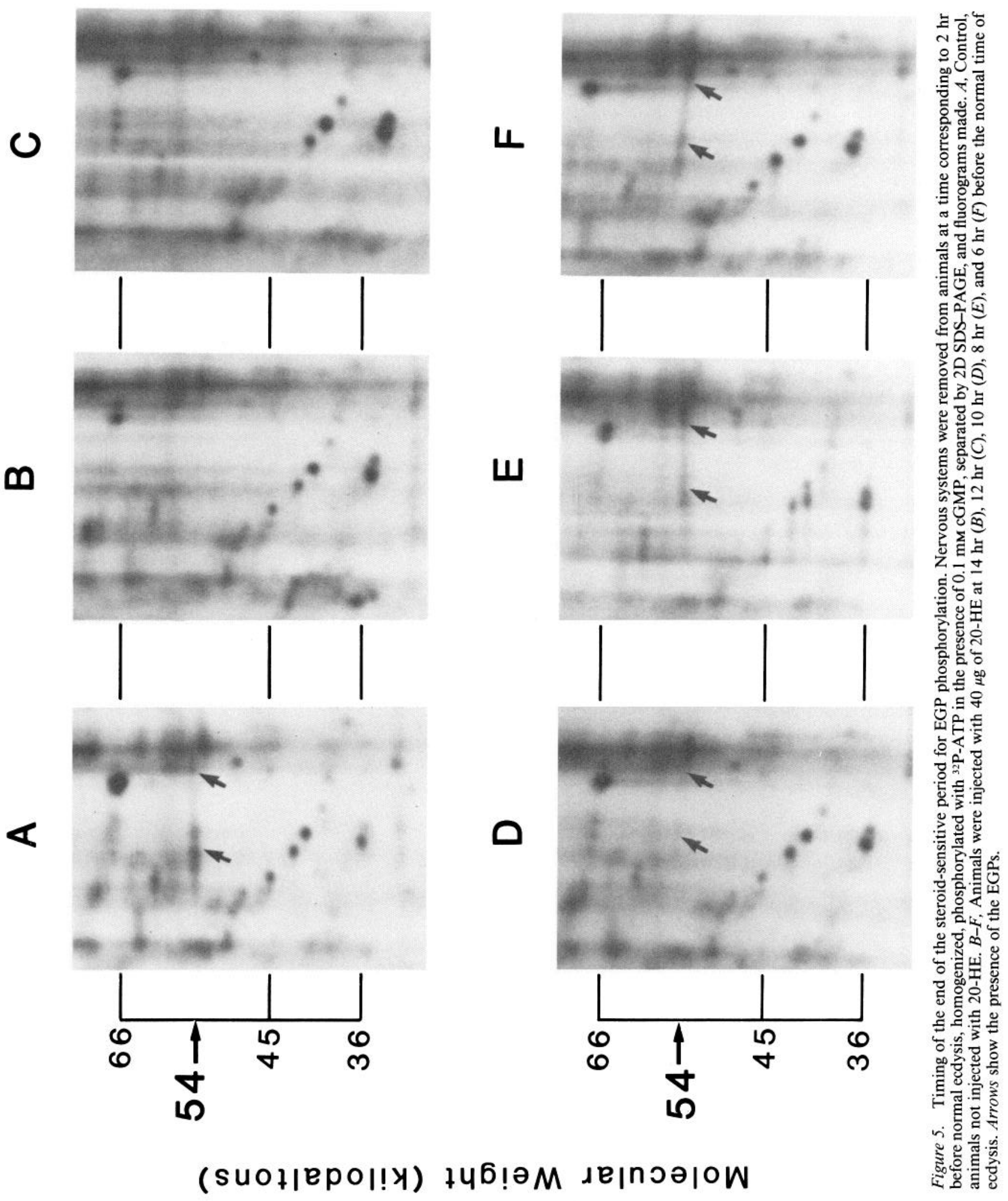
treated with $20-\mathrm{HE}$ at $-12 \mathrm{hr}$ showed no peak corresponding to the position of acidic EGP(EGP-A), whereas scans from animals treated with $20-\mathrm{HE}$ at $-10 \mathrm{hr}$ showed the level of incorporation of label to be $16 \%$ of that found when animals were not treated with 20 -HE. At later times, ecdysteroid treatment was essentially ineffective.

Injections of 20-HE $12 \mathrm{hr}$ before ecdysis or later no longer delayed the aquisition of behavioral sensitivity to $\mathrm{EH}$. The critical time after which $20-\mathrm{HE}$ no longer blocked the ability to phosphorylate the EGPs was similar and occurred 10-12 hr before ecdysis, approximately $4 \mathrm{hr}$ before the proteins could first be seen on fluorograms.

\section{Discussion}

Steroid hormones that modulate the way the CNS responds to peptides are relatively common in vertebrates. Two good examples are estrogen, whose actions regulate the response of the rat brain to LHRH, and oxytocin (Pfaff, 1973; Fahrbach et al., 1985). As yet, however, there is little understanding of the mechanism of this type of steroid action in the brain, so it has been necessary to turn to peripheral tissues for models in which to study these interactions. The oxytocin sensitivity of the myometrium in rats depends on the prior priming of the uterus with estrogen. In this case an important effect of the estrogen is to dramatically increase the number of oxytocin receptors (Soloff, 1985).

The interaction of ecdysteroids and EH in the insect CNS is a further example of such steroid-peptide interactions. EH acts directly on the CNS to release the stereotyped motor program used for ecdysis (Truman, 1978; Weeks and Truman, 1984). Throughout the life of the insect, it periodically becomes sensitive to EH, and on each occasion this sensitivity is immediately preceded by a major exposure to ecdysteroids (Truman et al., 1983).

The exposure of the CNS to 20-HE appears to regulate at least 2 factors in the cascade that mediates $\mathrm{EH}$ action. The first is the ability of EH to elevate cGMP (Morton and Truman, 1985); the second is the ability of the EGPs to be phosphorylated in vitro. In isolated abdomens, the CNS becomes capable of responding to $\mathrm{EH}$ with an increase in cGMP levels between 36 and $48 \mathrm{hr}$ after the end of an infusion with 20-HE (Morton and Truman, 1985). The ability to phosphorylate the EGPs is also dependent on ecdysteroids. Abdomen ligation $4 \mathrm{~d}$ before ecdysis completely prevented the ability to phosphorylate the EGPs. This prevention was due solely to the ligation's interrupting the flow of ecdysteroids from the anterior end of the insect. Infusion of such abdomens with $20-\mathrm{HE}$ resulted in the subsequent ability to phosphorylate the EGPs between 36 and $48 \mathrm{hr}$ after infusion. The amount of steroid delivered was critical. At the delivery rate we used ( $5.4 \mu \mathrm{g}$ of $20-\mathrm{HE} / \mathrm{hr})$, an infusion duration of greater than $3 \mathrm{hr}$ was required. One would expect to vary the critical duration depending on the rate of ecdysteroid delivery (Nijhout, 1974).

The time at which it was first possible to phosphorylate the EGPs relative to ecdysteroid exposure was similar in the isolated, infused abdomens to that in the intact animal. One difference, however, was that, in the intact animal, the ability to respond to $\mathrm{EH}$ with a cGMP increase occurred at least $12 \mathrm{hr}$ before the subsequent ability to phosphorylate the EGPs. In the infused preparations, by contrast, both capacities appeared between 36 and $48 \mathrm{hr}$ after the end of the infusion. Preliminary results (D. B. Morton and J. W. Truman, unpublished observations) suggest that the EH-stimulated CGMP increase requires only the rise in ecdysteroids and not their subsequent fall. The ability to phosphorylate the EGPs, however, requires both the rise and fall (see below). The shorter time interval between the rising and falling phases of the ecdysteroid titer in infused abdomens would then lead to a shorter interval between the cGMP response and the first appearance of the EGPs on fluorograms.

In the steroid-infusion experiments, the timing of the ability to phosphorylate the EGPs was correlated with the progression of other developmental markers associated with the pupal molt. Consequently, these experiments alone do not show whether the ecdysteroids are acting directly on the CNS or indirectly through effects on peripheral tissues. The in vitro experiments with the isolated abdominal CNS show that at least the last portion of ecdysteroid action on the CNS is indeed direct. The culture experiments also emphasize the fact that although ecdysteroid exposure is necessary for the subsequent phosphorylation of the EGPs, the steroid must then be removed before the proteins can be phosphorylated. A similar requirement for both the appearance and the removal of ecdysteroids is seen in the induction of the "late puffs" on the polytene chromosomes of Drosophila salivary glands (Ashburner et al., 1974), the activation of cuticle genes in the imaginal disks of Drosophila (Fristrom et al., 1982), and the activation of prophenoloxidase and the synthesis of dopa decarboxylase in the epidermis of Manduca larvae (Hiruma et al., 1985; Hiruma and Riddiford, 1986).

Injections of 20-HE at various times during the normal ecdysteroid decline (Fig. 4) show that events occur 10-12 hr before ecdysis, which prevents subsequent ecdysteroid exposure from inhibiting the ability to phosphorylate the EGPs. This time coincides with the low point in the ecdysteroid titer, which had declined to a value of $0.1 \mu \mathrm{g} 20-\mathrm{HE} \mathrm{Eq} / \mathrm{ml}$ (Truman et al., 1983). Although expressed as $20-\mathrm{HE} \mathrm{Eq} / \mathrm{ml}$, this value includes ecdysteroid metabolites other than $20-\mathrm{HE}$ that are recognized by the radioimmunoassay. Consequently, our results from the culture experiments, showing that levels of $20-\mathrm{HE}$ had to fall below $0.05 \mu \mathrm{g} / \mathrm{ml}$ before the EGPs could be phosphorylated, are likely to be within the physiological range.

In essentially all cases, ecdysteroids act via changes in transcription (see O'Connor, 1985). Accordingly, we suggest that the 20 -HE regulation of the ability to phosphorylate the EGPs involves the de novo synthesis of the EGPs themselves. Because the levels of the EGPs in the CNS of Manduca are too low to be detected directly, evidence for this hypothesis is only circumstantial. The time between the steroid critical period and the appearance of the EGPs on fluorograms is about $4 \mathrm{hr}$. This latency is consistent with their de novo synthesis. Furthermore, injecting animals with the protein synthesis inhibitor cycloheximide after the time of steroid independence shows that protein synthesis is necessary for the subsequent ability to phosphorylate the EGPs (D. B. Morton and J. W. Truman, unpublished observations). Although we have not shown directly that it is the EGPs that are synthesized, we consider this the most likely possibility.

The coincidence of the steroid critical period for both the appearance of the EGPs and EH responsiveness further supports our hypothesis that the regulation of the EGPs is the mechanism for the regulation of EH sensitivity. It is interesting to point out that although $20-\mathrm{HE}$ regulates $\mathrm{EH}$ sensitivity at at least 2 loci, they are independent of each other. At $16 \mathrm{hr}$ before ecdysis, the 
CNS has already established its steroid-sensitive cGMP system, but the EGPs are still sensitive to steroid manipulations.

Each step of a cyclic nucleotide-regulated cascade has the potential to be up- or down-regulated to alter the cellular response. Receptors can be up- and down-regulated either by synthesis/degradation or by receptor modification (see Hollenberg, 1985). The cyclic nucleotide-synthetic or breakdown pathway can also be altered, as with the regulation of guanylate cyclase activity by estrodiol in the ral brain (Amechi et al., 1985). Regulation of the protein kinase is seen in rat ovary (Richards and Rolfes, 1980), grasshopper epidermis (Vardanis, 1982), and Drosophila brain (Willmund, 1986). As far as we are aware, the present study is the only one that describes regulation of the substrate phosphoproteins as a means of altering the responsiveness of a system. Furthermore, this system is regulated in at least 2 steps. The first is the cGMP accumulation step, which occurs either by means of regulation of the receptor or of the cGMP synthesis/breakdown pathway (Morton and Truman, 1985), which renders the system partially competent to respond to EH. At pupal ecdysis, full responsivity seems to be provided by the appearance of the EGPs, possibly by their de novo synthesis. Both of these steps are regulated by $20-\mathrm{HE}$.

\section{References}

Amechi, O. A., P. J. Butterworth, and P. J. Thomas (1985) Effects of gonadal steroids on guanylate cyclase activity in the developing and adult brain. Brain Res. 342: 158-161.

Ashburner, M., C. Chihara, P. Meltzer, and G. Richards (1974) Temporal control of puffing activity in polytene chromosomes. Cold Spring Harbor Symp. Quant. Biol. 38: 655-662.

Bell, R. A., and F. A. Joachim (1978) Techniques for rearing laboratory colonies of tobacco hornworms and pink bollworms. Ann. Entomol. Soc. Am. 69: 365-373.

Bollenbacher, W. E., S. L. Smith, W. Goodman, and L. I. Gilbert (1981) Ecdysteroid titer during larval-pupal-adult development of the tobacco hornworm, Manduca sexta. Gen. Comp. Endocrinol. 44: 302306.

Fahrbach, S. E., J. I. Morrell, and D. W. Pfaff (1985) Role of oxytocin in the onset of estrogen facilitated maternal behavior. In Oxytocin Clinical and Laboratory Studies, J. A. Amico and A. G. Robertson, eds., pp. 372-388, Elsevier, New York.

Fristrom, J. W., J. Doctor, D. K. Fristrom, W. R. Logan, and D. J. Silvert (1982) The formation of the pupal cuticle by Drosophila imaginal discs in vitro. Dev. Biol. 91: 337-350.

Hiruma, K., and L. M. Riddiford (1986) Inhibition of dopa decarboxylase synthesis by 20 -hydroxyecdysone during the last larval molt of Manduca sexta. Insect Biochem. 16: 225-231.

Hiruma, K., L. M. Riddiford, T. L. Hopkins, and T. D. Morgan (1985) Roles of dopa decarboxylase and phenoloxidase in the melanisation of the tobacco hormworm and their control by 20 -hydroxyecdysone. J. Comp. Physiol. B 155: 659-669.

Hollenberg, M. D. (1985) Examples of homeospecific and heterospecific receptor regulation. Trends Pharmacol. Sci. 6: 242-245.

Laemmli, U. K. (1970) Cleavage of structural proteins during the assembly of the head of the bacteriophage T4. Nature 227: 680-685.
Meltzer, Y. L. (1971) Hormonal and attractant pesticide technology. Noyes Data Corp., Park Ridge, NJ.

Merril, C. R., D. Goldman, and M. L. Van Keuran (1983) Silver staining methods for polyacrilamide gel electrophoresis. Methods Enzymol. 96: 230-239.

Morton, D. B., and J. W. Truman (1985) Steroid regulation of the peptide-mediated increase in cyclic GMP in the nervous system of the hawkmoth, Manduca sexta. J. Comp. Physiol. A 157: 423-432.

Morton, D. B., and J. W. Truman (1986) Substrate phosphoprotein availability regulates eclosion hormone sensitivity in an insect CNS. Nature 323: 264-267.

Morton, D. B., and J. W. Truman (1988) The EGPs: The eclosion hormone and cyclic GMP-regulated phosphoproteins. I. Appearance and partial characterization in the CNS of Manduca sexta. J. Neurosci. 81: 1326-1337.

Nijhout, H. F. (1974) The role of ecdysone in pupation of Manduca sexta. J. Insect Physiol. 22: 453-463.

O'Connor, J. D. (1985) Ecdysteroid action at the molecular level. In Comprehensive Insect Physiology, Biochemistry and Pharmacology, vol. 8: Endocrinology II, G. A. Kerkut and L. A. Gilbert, eds., pp. 85-98, Pergamon, New York.

O'Farrell, P. Z., H. M. Goodman, and P. H. O'Farrell (1977) High resolution two-dimensional electrophoresis of basic as well as acidic proteins. Cell 12: 1133-1142.

Pfaff, D. W. (1973) Luteinizing hormone-releasing factor potentiates lordosis behavior in hypophysectomized ovariectomized female rats. Science 182: 1148-1149.

Reynolds, S. E., P. H. Taghert, and J. W. Truman (1979) Eclosion hormone and bursicon titres and the onset of hormonal responsiveness during the last day of adult development in Manduca sexta (L) J. Exp. Biol. 78: 77-86.

Richards, J. S., and A. I. Rolfes (1980) Hormonal regulation of cyclic AMP binding to specific receptor proteins in rat ovarian follicles. J. Biol. Chem. 255: 5481-5489.

Riddiford, L. M. (1985) Hormone action at the cellular level. In Comprehensive Insect Physiology, Biochemistry and Pharmacology, vol. 8 . Endocrinology II, G. A. Kerkut and L. A. Gilbert, eds., pp. 37-84, Pergamon, New York.

Soloff, M. S. (1985) Oxytocin receptors. In Neurosecretion and the Biology of Neuropeptides, H. Kobayashi et al., eds., pp. 267-276, Japan Science Society, Springer-Verlag, Tokyo.

Truman, J. W. (1978) Hormonal release of stereotyped motorprograms from the isolated nervous system of the Cecropia silkmoth. J. Exp. Biol. 74: 151-174.

Truman, J. W., P. H. Taghert, and S. E. Reynolds (1980) Physiology of pupal ecdysis in the tobacco hornworm, Manduca sexta. I. Evidence for control by eclosion hormone. J. Exp. Biol. 88: 327-337.

Truman, J. W., D. B. Rountree, S. E. Reiss, and L. M. Schwartz (1983) Ecdysteroids regulate the release and action of eclosion hormone in the tobacco hornworm, Manduca sexta (L). J. Insect Physiol. 29: 895900 .

Vardanis, A. (1982) An epidermal cyclic nucleotide dependent protein kinase with high activity at ecdysis. Insect Biochem. 12: 399-403.

Weeks, J. C., and J. W. Truman (1984) Neural organization of peptideactivated behaviors during the metamorphosis of Manduca sexta. I. Conservation of the peristalsis motor pattern at the larval-pupal transformation. J. Comp. Physiol. 155: 407-422.

Willmund, R. (1986) Longlasting modulation of protein kinase activity in the brain of Drosophila melanogaster induced by visual adaptation. J. Insect. Physiol. 32: 1-8. 